\title{
Isolation of Brain Tumor Segment using HMGMM
}

\author{
T.Selva Rani \\ P.G student \\ Anna University, Tirunelveli \\ Tirunelveli, 627005, India
}

\author{
K.Usha Kingsly Devi \\ Lecturer/ECE Department \\ Anna University, Tirunelveli \\ Tirunelveli, 627005, India
}

\begin{abstract}
Segmentation of images holds an important position in the area of image processing. It becomes more important while typically dealing with medical images, magnetic resonance (MR) imaging offers more accurate information for medical examination than other medical images such as X-ray, ultrasonic and CT images. Tumor segmentation from MRI data is an important but time consuming task performed manually by medical experts when compared with modern day's high speed computing machines which enable us to visually observe the volume and location of unwanted tissues.One of the reasons behind the inferior segmentation efficiency is the presence of artifacts in the MR images. One such artifact is the extracranial tissues (skull). These extracranial tissues often interfere with the normal tissues during segmentation that accounts for the inferior segmentation efficiency. This paper deals with an efficient segmentation algorithm for extracting brain tumors in magnetic resonance images using hidden Markov Gauss Mixture Model (HMGMM) with Genetic algorithm (GA). HMGMMs incorporate supervised learning, fitting the observation probability distribution given by each class using Gaussian mixture model. The GA and Expectation Maximization (EM) algorithms are used to obtain an HMM model with optimized number of states in the HMM models and its model parameters brain tumor extraction.
\end{abstract}

Keywords: Gaussian Mixture Model, Hidden Markov Model, Expectation Maximization algorithm, Genetic algorithm.

\section{INTRODUCTION}

Brain tumours remain one of the most difficult tumors to be treated, as they often attack areas where the treatment of choice namely the total tumour resection is virtually impossible. Correct decisions concerning surgery, radiotherapy and chemotherapy planning depend firmly on the accuracy of tumor segmentation performed by a specialized radiologist. This work done manually by the radiologist is a tedious and time consuming task. The aim of this paper is to create an algorithm that may be an extremely helpful tool for radiologists assisting them in brain tumor segmentation in MR images.

Magnetic Resonance Imaging (MRI) is an advanced medical imaging technique used to produce high resolution images of the parts contained in the human body.MRI imaging is often used when treating brain tumors. These high resolution images are used to examine human brain development and discover abnormalities. Nowadays there are several methodologies for classifying MR images. Among all medical image processing, image segmentation is initial and important work, for example, quantification of specified area must based on accurate segmentation.
For Segmentation, this paper uses Gauss Mixture Models (GMM) and it has been a popular tool for fitting smooth densities to data in statistics and statistical signal processing. The Gaussian probability (pdf) maximizes the differential entropy and the worst high-rate distortion rate trade off given the mean and the covariance [2], [4]. To design a GMM, employ a clustering approach using the k-Means algorithm. Accurate classification of GMM does not necessarily coincide with clear segmentation since the latter requires smooth boundaries between classes and better clustering for the same classes. Thus, additional spatial modeling is required for image segmentation. Among many context-dependent image segmentation algorithms, one popular method is based on Hidden Markov Models (HMM).

HMM is an important statistical modeling for automatic image segmentation. HMM can determine the statistics of variation from state to state. Nevertheless, many HMMrelated researches are still in progress. One of the main researches in the HMM research is to find a good HMM to segment an image. The major problem exist in this research is, how to optimize the model parameters. Many successful heuristic algorithms such as the Expectation - Maximization (EM) algorithms and the gradient methods are developed for the optimization of the HMM model parameters.

Genetic algorithm (GA) is a stochastic search method that can perform global search within the defined searching space and hopefully obtains the global optimal solution. Previously, Kwong et al. have applied the GA for HMGMM-based speech recognition \& web information extraction [3],[7]. GAHMGMM training gave better quality of solutions than the Expectation- Maximization algorithm by optimizing the HMM model parameters for the HMM training. GAHMGMM training is used to determine the optimal number of states for an image and also optimizes the model parameters in a single step.

The paper is organized as follows. In section 2,related work is explained briefly. Section 3 describes proposed work for image segmentation. In section 4, experimental results are illustrated. Finally, the conclusion is made in section 5.

\section{RELATED WORK}

A.P.Dempster, N.M.Lairad and D.B.Rubin (1977) introduced a broadly applicable algorithm for computing maximum likelihood estimates from incomplete data is presented at various levels of generality on Maximum likelihood from incomplete data via the EM algorithm [1]. The resultant EMalgorithm consists of "back and forth" least-squares calculations on the cross-products matrix of all variables (with the M-step supplemented in cases of special restrictions on R). This method is quite straightforward and can handle many cases using only familiar computations. 
Image classification by 2D HMM was introduced by J. Li, A. Najmi and R.M. Gray (2000) [2]. For block-based classification, an image is divided into blocks, and a feature vector is formed for each block by grouping statistics extracted from the block. Conventional block-based classification algorithms decide the class of a block by examining only the feature vector of this block and ignoring context information. In order to improve classification by context, an algorithm is proposed that models images by two dimensional (2-D) hidden Markov models (HMM's). The HMM parameters are estimated by the EM algorithm. To classify an image, the classes with maximum a posteriori probability are searched jointly for all the blocks. The 2-D model provides a structured way to incorporate context information into classification. As the model is 2-D, computational complexity is an important issue.

K. Pyun, et al.(2007) proposed a segmentation method combining GMVQ and 2-D hidden Markov modeling [4]. The problem $\mathrm{w}$ as formulated on the basis of Bayesian statistics. The context information using a 2-D hidden Markov model was incorporated to cluster the homogeneous classes. In order to improve segmentation by context, they devised an algorithm that models images by combining GMVQ and the MBP model to produce a 2-D noncausal HMGMM. The stochastic EM algorithm was applied to optimize the MAP hidden states of the HMGMM of the image. This approach was used to identify man-made regions in aerial images and to segment multiclass composite textures.

Jiyi Xiao, Lamei Zou and Chuanqi Lia introduced a new training method based on GA and Baum-Welch algorithms to obtain an HMM model with optimized number of states in the HMM models and its model parameters for web information extraction [3]. This method is not only able to overcome the shortcomings of the slow convergence speed of the HMM approach. In addition, this method also finds better number of states in the HMM topology as well as its model parameters. From this experiments with the 2100 webs extracted from our corpus, our method is able to find the optimal states in all cases. In addition, the HMM trained by GAHMM training have higher values of precision and recall than the HMM trained by the Baum-Welch algorithm.

\section{THE PROPOSED WORK FOR TUMOUR SEGMENTATION}

\subsection{Outline of the Algorithm}

An outline of the algorithm is as follows.

a) Remove skull of the MRI of brain tumor using skull stripping algorithm [8].

b) Select the number of states.

c) Apply k-means clustering algorithm.

d) Estimate GMM parameters based on k-means clustering.

e) Optimize HMM parameters using GA and Expectation Maximization algorithm.

f) Searching the set of classes with maximum a posteriori probability (MAP).

Gauss mixture models (GMMs) is applied to estimate the density of the source. An important problem in Gauss mixture modeling is the estimation of the means and covariance of the Gaussian components. The resulting GMM is the collection of the probabilities of occurrence, means, and variances, which describes a single image. The estimate of the mean and covariance matrix given one observation of is more challenging to compute. To estimate the parameters of Gauss Mixture, this paper uses K-Means clustering method [9].

Various steps in the K-Means algorithm are as follows:

1. Initialize the centroids for $\mathrm{N}$ states.

2. Repeat the following steps until the cluster labels of the image do not change anymore.

3. Cluster the points based on distance of their intensities from the centroid intensities.

$$
C^{(i)}=\operatorname{argmin}\left\|x^{(i)}-\mu_{j}\right\|^{2}
$$

4. Compute the new centroid for each of the clusters.

$$
\mu_{i}=\frac{\sum_{i=1}^{m}\left\{c_{(i)}=j\right\} x^{(i)}}{\sum_{i=1}^{m}\left\{c_{i}=j\right\}}
$$

Where $\mathrm{N}$ is a parameter of the algorithm (the number of clusters to be found), $i$ iterates over the all the intensities, $j$ iterates over all the centroids and $\mu_{i}$ are the centroid intensities.

\subsection{HMM}

The formal specifications of HMM are completely characterized by the following model parameters [3].

1. $N$, the number of states in the model.

2. $M$, the number of mixtures in the random function.

3. $A$, the state transition probability distribution. $A=\{a i j\}$, where $\mathrm{a}_{i j}$ is the transition probability from state $i$ to state $j$. That is

$a_{i j}=P\left[q_{t+1}=j \mid q_{t}=i\right] 1 \leq i, j \leq N$

4. $\pi$, the initial state distribution, $\Pi=\left\{\Pi_{i}\right\}$, Where

$$
\Pi_{i}=P\left[q_{i}=i\right], \quad 1 \leq i \leq N
$$

\subsection{Segmenting with HMGMM}

The proposed HMGMM is defined as follows. Given the hidden states $\mathrm{Z}=\left\{Z_{\mathrm{ij}}\right\}$, the observed images $\mathrm{X}=\left\{\mathrm{X}_{\mathrm{ij}}\right\}$ are conditionally independent Gauss mixture distributions estimated by

$$
P(X=x \mid Z=z)=\prod_{(i, j) \in \mathbb{R}} P\left(X_{i j}=x_{i j} \mid Z_{i j}=z_{i j}\right)
$$

The HMGMM is applied to MRI of brain tumor in order to segment the tumour area. The only parameter $\mathrm{p}$ is unknown and estimates it jointly with the true segmentation. Use a maximum a posteriori (MAP) estimate for the true segmentation and compute the approximate maximum likelihood estimator of often referred to as the hyper parameter [4]. In the HMGMM, the MAP estimate of Z, say $\mathrm{Z}^{*}$, becomes

$$
\begin{aligned}
& \mathrm{Z}^{*}=\operatorname{argmax} \log \mathrm{P}(\mathrm{Z}=\mathrm{z} \mid \mathrm{X}) \\
& \quad=\operatorname{argmax}\{\log \mathrm{P}(\mathrm{X} \mid \mathrm{Z}=\mathrm{z})+\log \mathrm{P}(\mathrm{Z} \mid \mathrm{z})\} .
\end{aligned}
$$

The first term in the MAP (Maximum a Posteriori), log $\mathrm{P}(\mathrm{X} \mid \mathrm{Z}=\mathrm{Z})$ is modeled by the GMM. This step can be viewed 
as a training stage in supervised learning, whereas $\mathrm{P}(\mathrm{Z} \mid \mathrm{z})$ (the probability of spatial correlations), which is the second term in the MAP, is evaluated in a testing stage. When a new image of interest comes for testing, the unknown parameter in MAP equation is evaluated each time, producing a different parameter for each run. This paper uses the EM procedures for estimating the parameters [5].

\subsection{Genetic Algorithm}

The genetic algorithm is a searching process based on the laws of natural selection and genetics. It emulates the individuals in the natural environment that the natural selection mechanism makes the stronger individuals likely winners in the competing environment.

Genetic algorithms are one of the best ways to solve a problem for which little is known. They are a very general algorithm and so will work well in any search space. All you need to know is what you need the solution to be able to do well, and a genetic algorithm will be able to create a high quality solution. Genetic algorithms use the principles of selection and evolution to produce several solutions to a given problem.

Generally, a simple GA cycle consists of four operations: Fitness evaluation, selection, genetic operations, and replacement. In a simple GA cycle, there exists a population pool of chromosomes. The chromosomes are the encoded form of the potential solutions and all GA operations except the fitness evaluation to be performed with this form of solutions. Initially, the population is generated randomly and the fitness values of all the chromosomes are evaluated by calculating the objective function in the decoded form of chromosomes. After the initialization of the population pool, the GA evolution cycle is begun. At the beginning of each generation, the mating pool is formed by selecting some chromosomes from the population. This pool of chromosomes is used as the parents for the genetic operations to generate the offspring or the subpopulation. The fitness values of the offspring are also evaluated. At the end of the generation, some chromosomes in the population will be replaced by the offspring according to the replacement scheme [3]

The above generation is repeated until the termination criterion is met. By emulating the natural selection and genetic operations, this process will hopefully leave the best chromosomes or the highly optimized solutions to the problem in the final population. It is noted that the genetic operations in GA provide the global searching capability to the problem. Therefore, the HMM training can escape from the initial guess and find the optimal solution if we applied the GA to the training process. The outline of the genetic algorithm is as follows.

- Start with a randomly population of chromosomes.

- Calculate the fitness function of each chromosome in the population.

- Apply selection, crossover and mutation to the population, and then a new population is generated.

- Make a judgment whether the algorithm satisfies terminating condition. If it does not, go to the second step; if it does, the GA is terminated and the optimal value for image segmentation is obtained.

\section{1) Encoding}

The chromosome is usually expressed in a string of elements and each element of which is called a gene. According to the problem specifications, a gene can be defined as the type of binary, real number, or other forms. Bit-string encoding is the most classic approach used by GA researchers due to its simplicity and traceability. However, the basic data type of the HMM element is real number, so this experiment use real number string instead of bit-string as the representation of the chromosomes in GA.

\section{2) Fitness evaluation}

The fitness evaluation is a mechanism used to determine the confidence level of the optimized solutions to the problem. Usually, there is a fitness value associated with each chromosome. A higher fitness value means that the chromosome or solution is more optimized to the problem while a lower value of fitness indicates a less optimized chromosome. In our GA, the fitness values are the results of the objective function. The likelihood $\mathrm{P}[\mathrm{X} \mid \mathrm{z}]$ is an appropriate criterion used in the objective function to determine the quality of the chromosomes. The probability $\mathrm{P}[\mathrm{X} \mid \mathrm{z}]$ is calculated by maximum likelihood method .

\section{3) Selection}

The selection mechanism is to select the parent chromosomes from the population and forms the mating pool. The selection mechanism emulates the survival of the fittest mechanism in nature. It is expected that a fitter chromosome receives a higher number of offspring and thus has a higher chance of surviving in the subsequent evolution while the weaker chromosomes will die eventually. The Roulette wheel selections, one of the most common easy way to implement selection mechanism. A virtual wheel is used in this selection mechanism. Each chromosome in the population is associated with a sector in the virtual wheel. According to the fitness value of the chromosome, the sector will have a larger area when the corresponding chromosome has a better fitness value while a lower fitness value will lead to a smaller sector. In order to implement the Roulette wheel selection for GA, use $\mathrm{Eq}(7)$ to normalize the fitness values of the chromosomes.

$$
p_{i}=F_{i} / \sum_{i=1}^{m} F_{i}, i=1,2, \ldots M
$$

Where $\mathrm{Pi}$ is the normalized fitness value of Mth chromosome in the population and $\mathrm{Fi}$ is the fitness value of chromosome in the population.

\section{4) Crossover}

Crossover operator is a recombination operator that combines subparts of the parents to produce offspring that contain some parts of both parent genetic materials. The selected parents most likely are the fitter chromosomes. Therefore, it can be seen that this operator is aimed to combine the optimized genetic materials in the parents together and to produce more optimized offspring. In this operator, two parents will be selected from the population pool based on the Roulette wheel mechanism.

\section{5) Mutation}

Mutation adds variations of model parameters into chromosomes. It provides the global searching capability to GA by randomly altering the values of genes in the chromosomes. It recovers the lost information in the initialization phase of GA and makes GA escape from the initial model parameters and to find the optimal model 
parameter set. According to an alternation probability, each model parameter in the parent may or may not be altered by this genetic operator. Before the modification of a model parameter, the mutation rate will be compared with a randomly generated probability to test if the mutation rate is larger than or equal to the randomly generated probability.

\section{6) Image segmentation}

GA-HMM is used for brain tumor extraction by formulating a model in the following way: each state is associated with a class that is needed to extract, such as tumor tissue, normal tissue and background area. Thus class-specific distribution and the state transition probabilities from training data by GAHMM hybrid operations are studied. In order to label a tumorous area with classes, and recover the most-likely state sequence with the Maximum a Posteriori (MAP).

\section{EXPERIMENTAL RESULTS}

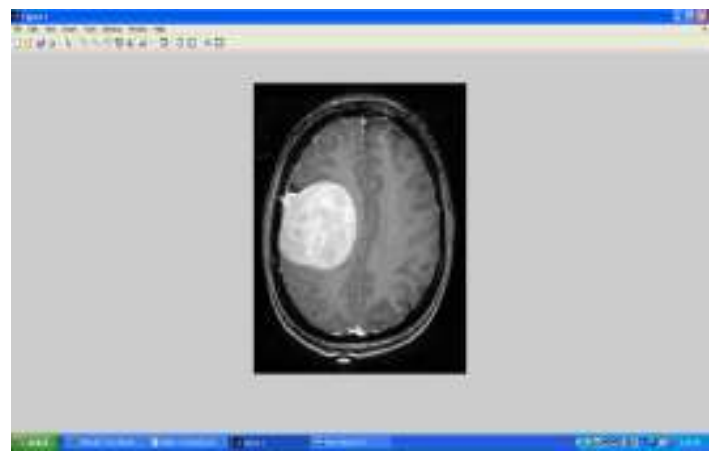

Fig1: Input image

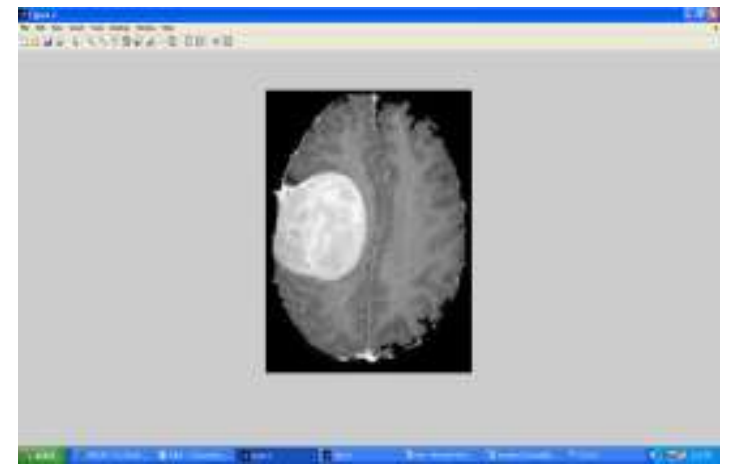

Fig 2: After skull stripping

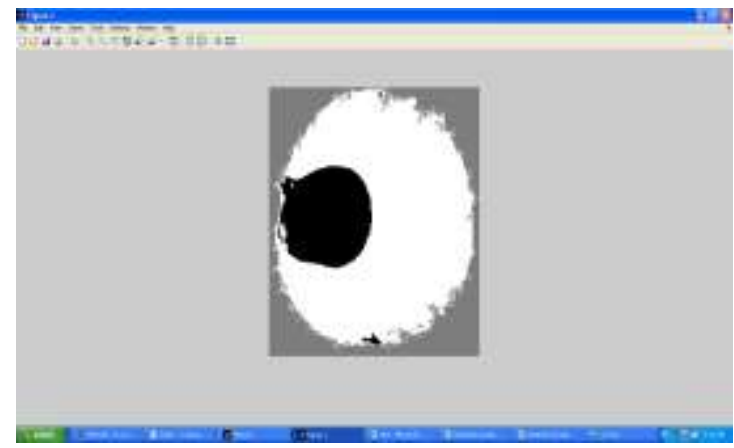

Fig 3: segmented image using HMGMM

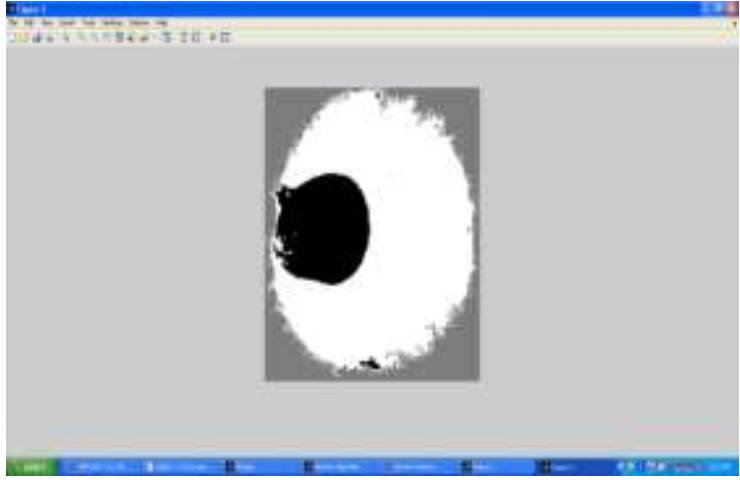

Fig 4: segmented image using HMGMM with GA

Figure1 shows the input image of size 226X226. Black represents tumor area, white represents normal brain area and gray color represents background of an image. HMGMM with GA gives good results for most of the iterations and achieve better segmentation.

The following GA parameters are used for this experiment. Population size: 50

Crossover rate: 0.6

Mutation rate: 0.01

\section{CONCLUSION}

This paper presented a new HMM training method based on genetic algorithms for brain tumour extraction. This method finds good HMM topology as well as its model parameters. This method is able to find the optimal states in all cases. The HMM trained by GA-HMGMM method are more optimized than the HMM trained by the Expectation Maximization (EM) algorithm. It can be concluded that the model parameters of the HMM are optimized in one single step. From the experimental results, the genetic algorithm can find the optimal number of states even in the case of uneven initial distribution of the number of occurrences of the chromosomes with different number of states. We also find that GA is a very easy to- use optimization algorithm. By using a very simple genetic operator, the GA can find the optimal number of states in an image efficiently.

\section{REFERENCES}

[1] A. P. Dempster, N. M. Laird, and D. B. Rubin, "Maximum likelihood from incomplete data via the EM algorithm," J. Roy. Statist.Soc,vol. 39, no.1, pp. 1-21, 1977.

[2] J. Li, A. Najmi, and R. M. Gray, "Image classification by a two-dimensional hidden Markov model," IEEE Trans. Signal Process, vol. 48, no. 2, pp. 517-533, Feb. 2000.

[3] J.Xiao, L.Zou, and C.Li,"optimization of Hidden Markov Model by a Genetic algorithm for web information extraction" University of south China, Hengyang 421001 .

[4] K. Pyun, J. Lim, C. S. Won, and R. M. Gray, "Image segmentation using hidden Markov gauss mixture models," IEEE Trans. Image Process., vol. 16, no. 7, pp. 1902-1911,Jul. 2007.

[5] L. R. Rabiner. A tutorial on hidden Markov models and selected applications in speech recognition. Proc. IEEE, 77(2):257-285, February 1989. 
[6] Paweł Szwarc,"Segmentation of Brain Tumors in MR Images," Silesian University of Technology, 2007.

[7] Q.Y. Hong and S. Kwong, A genetic classification method for speaker recognition. Engineering Applications of Artificial intelligence, 18:13-19, 2005.

[8] Shanthi K. J, Dr. M. Sasi Kumar "Skull Stripping and AutomaticSegmentation of Brain MRI Using Seed Growth and Threshold Techniques" International Conference on Intelligent and Advanced Systems 2007
[9] Suman Tatiraju, Avi Mehta "Image Segmentation using kmeans clustering, EM and Normalized Cuts", University Of California - Irvine

[10] Terrence Chen, and Thomas S. Huang, "Region Based Hidden Markov Random Field Model for Brain MR Image Segmentation," World Academy of Science, Engineering and Technology 4, 2005. 